\title{
Prevalence of multimorbid degenerative lumbar spinal stenosis with knee or hip osteoarthritis: a systematic review and meta-analysis
}

James J. Young ${ }^{1,2,3^{*}}$, Rikke Krüger Jensen ${ }^{1,4}$, Jan Hartvigsen ${ }^{1,4}$, Ewa M. Roos ${ }^{1}$, Carlo Ammendolia ${ }^{5,6}$ and Carsten Bogh Juhl $1^{1,7}$

\begin{abstract}
Background: Musculoskeletal multimorbidity is common and coexisting lumbar spinal stenosis (LSS) with knee or hip osteoarthritis (OA) has been reported. The aim of this review was to report the prevalence of multimorbid degenerative LSS with knee or hip OA based on clinical and/or imaging case definitions.

Methods: Literature searches were performed in MEDLINE, EMBASE, CENTRAL, and CINAHL up to May 2021. Studies involving adults with cross-sectional data to estimate the prevalence of co-occurring LSS with knee or hip OA were included. Study selection, data extraction, and risk of bias assessment were performed independently by two reviewers. Results were stratified according to index and comorbid condition, and by case definitions (imaging, clinical, and combined).
\end{abstract}

Results: Ten studies from five countries out of 3891 citations met the inclusion criteria. Sample sizes ranged from 44 to 2,857,999 (median 230) and the mean age in the included studies range from 61 to 73 years (median 66 years). All studies were from secondary care or mixed settings. Nine studies used a combined definition of LSS and one used a clinical definition. Imaging, clinical, and combined case definitions of knee and hip OA were used. The prevalence of multimorbid LSS and knee or hip OA ranged from 0 to 54\%, depending on the specified index condition and case definitions used. Six studies each provided prevalence data for index LSS and comorbid knee OA (prevalence range: 5 to $41 \%$ ) and comorbid hip OA (prevalence range: 2 to 35\%). Two studies provided prevalence data for index knee OA and comorbid LSS (prevalence range 17 to 54\%). No studies reporting prevalence data for index hip OA and comorbid LSS were found. Few studies used comparable case definitions and all but one study were rated as high risk of bias.

Conclusions: There is evidence that multimorbid LSS with knee or hip OA occurs in people (0 to 54\%), although results are based on studies with high risk of bias and surgical populations. Variability in LSS and OA case definitions limit the comparability of studies and prevalence estimates should therefore be interpreted with caution.

Review registration: PROSPERO (CRD42020177759).

\footnotetext{
*Correspondence: jyoung@health.sdu.dk

${ }^{3}$ Centre for Muscle and Joint Health, Department of Sports Science

and Clinical Biomechanics, University of Southern Denmark, 55

Campusvej, DK-5230 Odense M, Denmark

Full list of author information is available at the end of the article
}

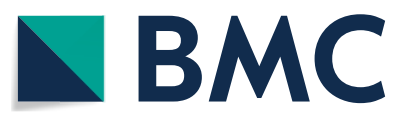

(c) The Author(s) 2022. Open Access This article is licensed under a Creative Commons Attribution 4.0 International License, which permits use, sharing, adaptation, distribution and reproduction in any medium or format, as long as you give appropriate credit to the original author(s) and the source, provide a link to the Creative Commons licence, and indicate if changes were made. The images or other third party material in this article are included in the article's Creative Commons licence, unless indicated otherwise in a credit line to the material. If material is not included in the article's Creative Commons licence and your intended use is not permitted by statutory regulation or exceeds the permitted use, you will need to obtain permission directly from the copyright holder. To view a copy of this licence, visit http://creativecommons.org/licenses/by/4.0/. The Creative Commons Public Domain Dedication waiver (http://creativeco mmons.org/publicdomain/zero/1.0/) applies to the data made available in this article, unless otherwise stated in a credit line to the data. 
Keywords: Lumbar spinal stenosis, Knee osteoarthritis, Hip osteoarthritis, Multimorbidity, Prevalence

\section{Background}

Multimorbidity (the co-existence of two or more chronic conditions) is an increasing health challenge as global populations continue to age [1-3]. Chronic musculoskeletal comorbidities are frequently reported as part of the multimorbid chronic disease profile [4] and increase the risk of developing other non-communicable diseases [5]. Multimorbidity must be considered in care models for musculoskeletal health [6] and older adults [7].

The prevalence of degenerative lumbar spinal stenosis (LSS) with knee or hip osteoarthritis (OA) - three of the most common chronic musculoskeletal conditions in the aging population - continue to rise [8-10]. Similar to knee and hip OA, LSS is most often a result of degenerative changes [11] and these diseases may co-exist in people with either index condition [12]. Supporting this hypothesis, a recent analysis from the Johnston County OA Project found $44 \%$ and $34 \%$ of people with imaging findings associated with lumbar spine OA also had image-defined knee and hip OA, respectively [13]. Clinical evidence also suggests lower extremity arthritis is common in people with LSS undergoing surgery $[14,15]$.

In both LSS and OA, radiological and clinical case definitions are used in prevalence estimates, and results differ according to the case definition employed $[9,16]$. No gold-standard case definition exists for either LSS or knee and hip OA, but definitions where both clinical and imaging findings are combined may be preferred since symptoms experienced by people with LSS and knee and hip OA often do not relate to the degree of structural change observed via imaging $[11,17]$.

Irrespective of criteria used, co-occurring disease is likely important in the clinical decision-making process. Multimorbidity in general is associated with a worse quality of life and future functional decline [18-20]. Musculoskeletal-specific multimorbidity is also associated with decreased health status metrics [21-23], particularly true in both low back pain [24] and multi-joint OA $[25,26]$. In people with LSS, the presence of comorbidities (including lower extremity arthritis) is associated with worse preoperative function [14], and comorbid knee and hip OA are associated with increased odds of poorer postoperative function [15]. Finally, people with LSS are less likely to attain a meaningful improvement following surgery when they also report additional symptomatic joints, including the knee and hip [27].

A comprehensive summary of available literature is needed to determine how frequently LSS and knee and hip OA coexist as a first step in improving clinical decision-making. The overall aim of this review was to report the prevalence of multimorbid degenerative LSS with knee or hip OA based on clinical and/or imaging case definitions.

\section{Methods}

The protocol for this review has been published [28] and registered in PROSPERO (CRD42020177759). This review was conducted according to the guidelines from the Cochrane Collaboration [29] and reported according to the Preferred Reporting Items for Systematic Reviews and Meta-Analysis (PRISMA) statement [30] (Additional file 1).

\section{Objectives}

This review had four objectives:

1. To estimate the prevalence of index LSS with comorbid knee OA based on imaging, clinical, and combined case definitions.

2. To estimate the prevalence of index knee OA with comorbid LSS based on imaging, clinical, and combined case definitions.

3. To estimate the prevalence of index LSS with comorbid hip OA based on imaging, clinical, and combined case definitions.

4. To estimate the prevalence of index hip OA with comorbid LSS based on imaging, clinical, and combined case definitions.

\section{Case definitions}

All case definitions for degenerative LSS and knee and hip OA were included and were broadly classified into three categories as imaging; clinical; and combined diagnoses encompassing both imaging and clinical diagnoses. The following diagnoses and clinical manifestations were included based upon study eligibility criteria: All imaging diagnoses based on radiographic, magnetic resonance imaging, or computerized tomography for the lumbar spine, knee, and hip; clinical diagnoses based on signs and symptoms of LSS, knee OA, and hip OA; all clinical manifestations of LSS (neurogenic claudication, radicular type, and mixed types) as they represent central, lateral, and combined central and lateral canal stenosis [31]. Patient self-reported diagnoses and medical chart reviews, including International Classification of Disease 
codes, were considered to be clinical diagnoses for both LSS and OA, unless explicitly stated otherwise. We considered surgical samples for LSS and/or OA (without explicit case definitions) to be combined diagnoses as it is unlikely that individuals would be offered surgery based upon imaging or clinical findings alone. For example, if a study included participants undergoing surgery for knee OA who were also evaluated for LSS by imaging, knee OA was designated as the index condition and assigned a combined case definition, whereas LSS was designated as the comorbid condition and assigned an imaging case definition.

\section{Search strategy}

The literature search was performed on May 03, 2021 in the following databases with no publication date or language limitation: MEDLINE, EMBASE, CENTRAL, and CINAHL. The search strategy was developed with a health sciences librarian based upon previous Cochrane reviews on LSS [32] and knee [33] and hip OA [34]. Search terms related to LBP were included in the LSS search concept to ensure potentially relevant literature was captured. The search was developed in MEDLINE and adapted to the other databases (Additional file 2).

Forward citation tracking of included studies was performed in Web of Science and reference lists were searched for additional relevant studies. The reference list from a recent review on LSS prevalence [9] was searched for relevant studies. PROSPERO was searched for relevant ongoing or completed systematic reviews. Additionally, scientific abstracts presented at the International Forum for Back and Neck Pain Research in Primary Care and the Osteoarthritis Research Society International World Congress between 2018 and 2020 were reviewed to identify publications potentially relevant to this review. Finally, LSS content experts known to the author team were also contacted to identify any known publications relevant to our research question.

\section{Eligibility criteria}

Studies were considered for inclusion if they: (1) were cross-sectional, cohort or case-control studies, or randomized controlled trials; (2) included adults 18 years or older; (3) assessed the prevalence of co-occurring LSS with knee and/or hip OA or present sufficient crosssectional data for estimating the prevalence (number of participants with LSS, number of participants with knee and/or hip OA, and total number of participants); and (4) were full-text papers published in English in peerreviewed journals. Studies were excluded if they: (1) included individuals with low back, knee, or hip pain due to other origins (e.g., fracture, tumour, inflammatory disease, infection, lumbar disc herniation); (2) were laboratory or cadaveric studies; (3) were conference abstracts; (4) included congenital or non-degenerative forms of LSS, without separate data on degenerative LSS; or (5) provided aggregate prevalence data for knee and hip OA separately.

\section{Study selection}

Records identified in the search strategy were screened using a two-stage process by two reviewers (JJY and RKJ) using a sensitive screening approach where any title or abstract mentioning any of the following were moved to full-text review: (1) LSS in isolation; (2) knee or hip OA and comorbidities; (3) knee or hip OA and LBP; or (4) prevalence of multiple musculoskeletal conditions. This approach was taken to mitigate the exclusion of relevant studies due to lack of reporting of information relevant to this review in the abstract. First, reviewers independently screened titles and abstracts against the eligibility criteria. Discrepancies were resolved through discussion.

Next, full-text versions of the remaining potentially relevant studies were independently screened. Discrepancies were resolved through discussion or independent review by a third reviewer (CBJ) when necessary. Reasons for exclusion of full-text articles were recorded. All references returned in the database search were managed using Endnote X9 (Clarivate Analytics, Philadelphia, USA) and Covidence systematic review software (Veritas Health Innovation, Melbourne, Australia). Absolute agreement and the Kappa coefficient [35] was calculated for both stages of screening using the Covidence feature.

\section{Data extraction}

The following information was extracted from all included papers by two independent reviewers (JJY and RKJ) using a standardized extraction form: first author, publication year, country, study design, population (LSS, knee OA, or hip OA), inclusion criteria, study setting, age, sex, case definitions of LSS and knee and/or hip OA, numerator and denominator for prevalence calculation, and items used in the risk of bias assessment.

\section{Risk of bias assessment}

Two reviewers (JJY and RKJ) independently assessed the risk of bias in all included studies using a modified version of the Risk of Bias Tool for Prevalence Studies [36]. Modifications made to the tool for the purposes of this study have been described in detail in the review protocol [28] (Additional file 3). In summary, we removed item 5 from the original tool as we assumed both clinical and imaging information can only be collected directly from participants. Further, items 6 and 7 (acceptability of case definition and validity/reliability of study instruments) were split into two questions to allow 
for individual ratings of LSS and OA definitions and measurement properties. Finally, we included an additional response option of "irrelevant" for item 9 because studies using imaging definitions are not impacted by recall bias. The modifications for this study followed the approach taken in two recent systematic reviews using the same tool $[9,24]$.

Individual items were rated as "Yes" for low risk of bias or "No" for high risk of bias or if there was insufficient information presented for adequate judgement. No specific quantitative thresholds were used to judge the overall risk of bias. Rather, a judgement on the internal and external validity taking into consideration the responses to each item on the modified tool was used to determine the overall risk of bias (low, moderate, high) by the two reviewers.

\section{Data management and synthesis}

Pooled prevalence estimates with $95 \%$ CI for each combination of LSS with knee or hip OA (based on case definitions) was performed using a random effects model. Due to the limited number of studies using comparable case definitions for LSS and knee and/or hip OA, only pooled estimates for combined LSS and co-occurring (i) clinical knee OA (ii) imaging knee OA, (iii) clinical hip OA, and (iv) imaging hip OA were calculated and are summarized. One study reporting a prevalence of $0 \%$ was artificially given a numerator of 0.001 . The low number of studies used in each of the pooled estimates prevented the calculation of heterogeneity statistics and no pre-planned meta-regression analyses [28] were performed. All statistical analyses were performed in Stata 16.1 (StataCorp LLC, College Station, USA).

\section{Results \\ Study selection}

The database search identified a total of 3891 citations with 307 additional citations identified through other sources (Additional file 1). After removal of duplicates, titles and abstracts of the remaining 2891 records were screened and 517 articles were deemed relevant for full-text screening. Absolute agreement between reviewers was $90 \%$ with a Kappa of 0.62 . After full-text screening, ten articles met the eligibility criteria for this review [37-46]. Two excluded articles provided prevalence data on LSS with comorbid knee and hip OA, but were excluded because separate estimates for knee and hip OA were not provided [47, 48]. Absolute agreement for full text screening was $98 \%$ with a Kappa of 0.68 . The study selection process is presented in Fig. 1.

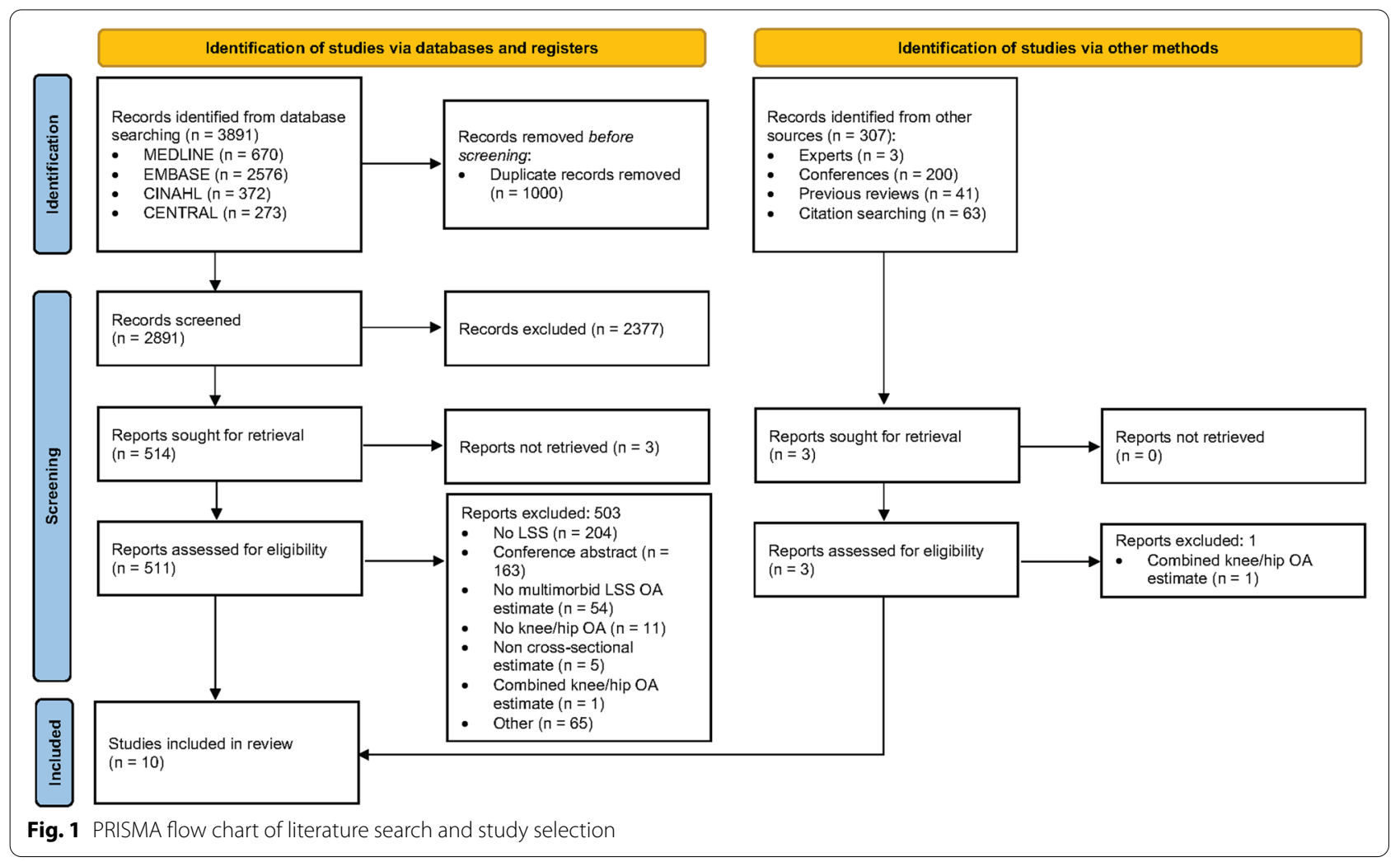




\section{Study characteristics and results}

No included studies had an objective of estimating the prevalence of multimorbid LSS with knee and/or hip OA. Sample sizes ranged from 44 to $2,857,999$ (median 230) with a median age of 66 years (range 61 - 73).

\section{LSS and comorbid knee OA}

Six studies estimating the prevalence of index LSS with comorbid knee OA were included [37, 39-41, 43, 45] (Table 1). Half of the studies were from Korea $(n=3)$ [39-41], with one study each from Japan [43], USA [45], and Switzerland [37]. Study designs were cross-sectional $(n=2)[40,41]$, cohort $(n=2)[37,39]$, case-control $(n=1)$ [43], and randomized controlled trial $(n=1)$ [45]. All studies were from secondary care settings with the exception of one study that included a mixed sample [45]. Sample sizes ranged from 44 to 641 (median 183). The proportion of females included ranged from 52 to $100 \%$ and the mean age ranged from 61 to 73 years (median 68). A combined case definition (imaging and clinical findings) was used in all studies for the index LSS condition. Combined $(n=1)$, clinical $(n=2)$, and imaging $(n=3)$ case definitions of comorbid knee OA were used (Table 1).

The single study ( $n=641)$ using a combined definition of LSS with a combined definition of knee OA found a prevalence of 5\% (95\% CI 4-7\%) [43]. The pooled prevalence in two studies $(n=860)$ using combined definitions of LSS with clinical definitions of knee OA was 25\% (95\% CI 22-27\%) [37, 45]. The pooled prevalence in three studies $(n=217)$ using combined definitions of LSS and imaging definitions of knee OA was $41 \%$ (95\% CI 35-48\%) [39-41] (Fig. 2).

\section{Knee OA and comorbid LSS}

Two studies estimating the prevalence of index knee OA with comorbid LSS were included [38, 42] (Table 1). One study each was from Korea [38] and India [42] with one study using a cross-sectional design [38] and one a cohort [42]. One study used a sample from a secondary care [42] and the other from a national health insurance database [38]. Sample sizes were 200 and 2,857,999, the proportion of females was 62 and $82 \%$, respectively and the mean ages were 64 and 66 years. Both studies used a combined case definition for the index knee OA condition. Combined $(n=1)$ and clinical $(n=1)$ case definitions of comorbid LSS were utilized (Table 1).

It was not possible to pool prevalence estimates of knee OA with comorbid LSS. The single study $(n=200)$ using a combined definition of knee OA and a combined definition of LSS reported a prevalence of 54\% (95\% CI 47-61\%)
[42] whereas the other $(n=2,857,999)$ using a combined definition of knee OA and a clinical definition of LSS reported a prevalence of $17 \%$ (95\% CI 17-17\%) [38].

\section{LSS and comorbid hip OA}

Six studies estimating the prevalence of index LSS with comorbid hip OA were included [37, 39, 41, 44-46] (Table 2). Studies were performed in Korea $(n=2)[39,41]$, USA $(n=2)[45,46]$, Japan [44], and Switzerland [37]. Study designs were primarily cohort $(n=4)[37,39,44,46]$ with one cross-sectional study [41] and one randomized controlled trial [45]. All included studies enrolled LSS patients from secondary care settings, except one which included LSS patients from mixed settings [45]. Study sample size ranged from 44 to 601 participants (median 226). The proportion of females ranged from 3 to $100 \%$ and mean age from 61 to 73 years (median 66; one study age not reported). All studies used a combined case definition for the index LSS condition. Combined $(n=1)$, clinical $(n=2)$, and imaging $(n=3)$ case definitions of comorbid hip OA were utilized (Table 2).

The single study ( $n=193)$ using a combined case definition of LSS with a combined definition of hip OA was $35 \%$ (95\% CI 29-42\%) [46]. The pooled prevalence in two studies $(n=860)$ using combined definitions of LSS and clinical definitions of hip OA was 17\% (95\% CI 14-19\%) $[37,45]$. The pooled prevalence in three studies $(n=570)$ using combined definitions of LSS and imaging definitions of hip OA was $2 \%$ (95\% CI 0-5\%) [39, 41, 44] (Fig. 3).

\section{Hip OA and comorbid LSS}

No studies estimating the prevalence of hip OA with comorbid LSS met our eligibility criteria.

\section{Risk of bias}

All included studies were judged as having a high risk of bias, with the exception of one study that had a moderate risk of bias [38] (Table 3). External validity items were generally rated as high risk of bias, as all but one study [38] did not include random samples representative of the target population and did not provide information on study response rates. Internal validity was also generally rated as having a high risk of bias. Most studies used an acceptable case definition for LSS and knee or hip OA, but with inadequate validity and reliability of the case definitions.

\section{Discussion}

The prevalence of multimorbid LSS and knee or hip OA ranged from 0 to $54 \%$, depending on the specified index condition and case definitions used. The majority of multimorbid prevalence estimates are derived from samples of participants with an index condition of LSS and 


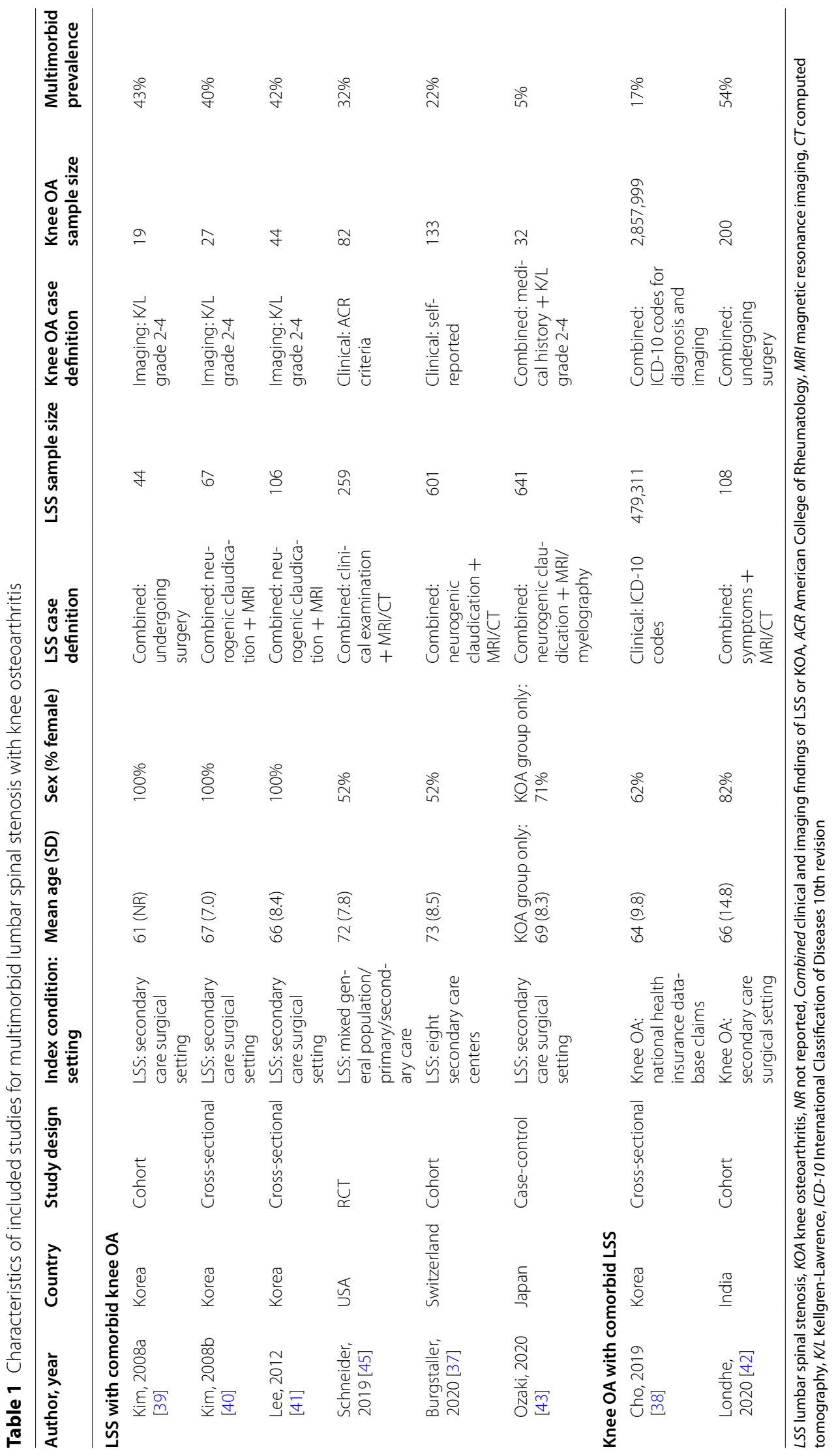




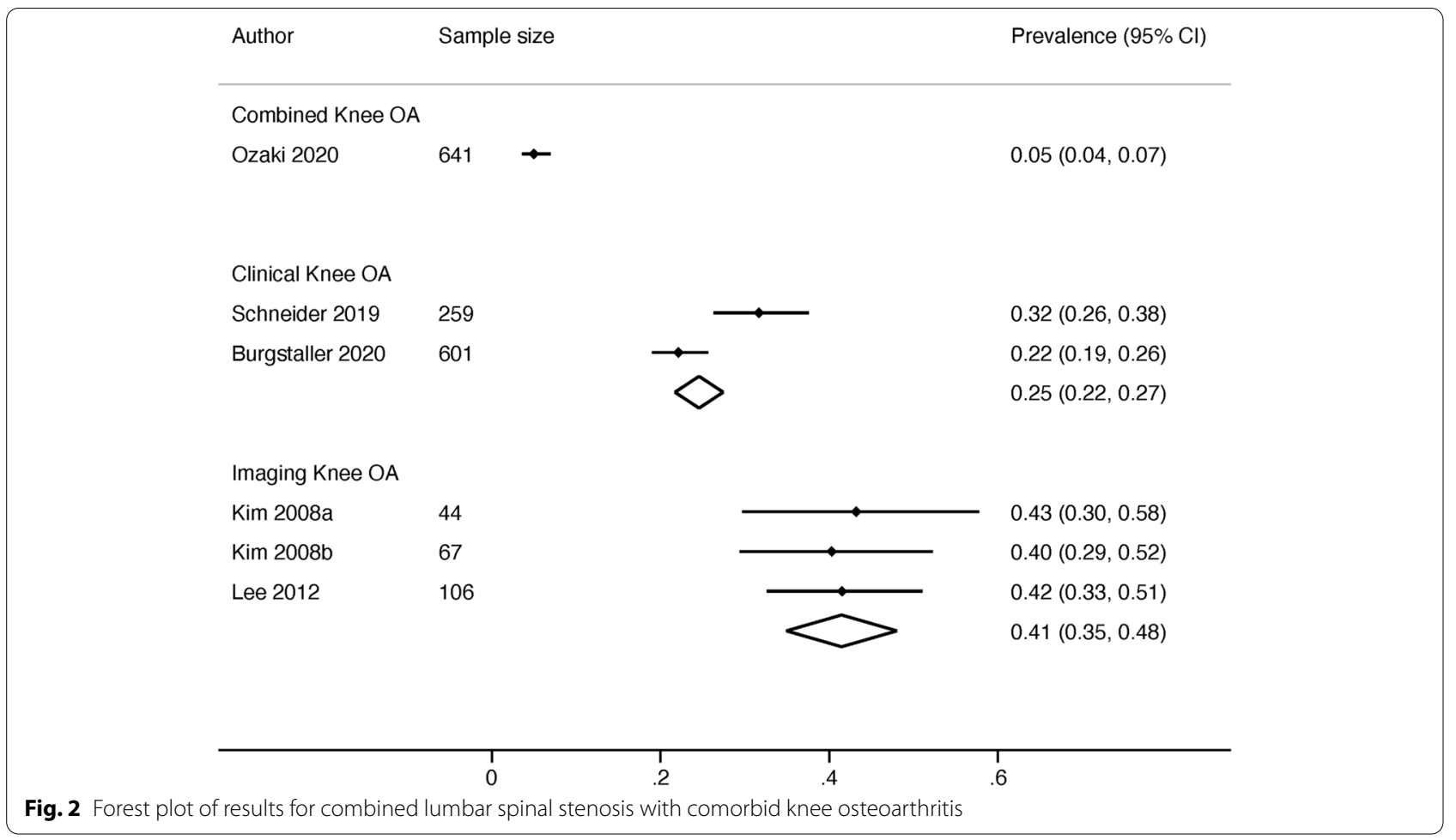

comorbid knee or hip OA. Few studies used the same combination of case definitions for the index and comorbid condition, but the comparable studies did show similar prevalence estimates. All but one included study were high risk of bias. Included prevalence estimates were primarily derived from surgical samples. However, in presenting the first pooled data and prevalence estimates for multimorbid LSS with knee or hip OA this study may be of value to those planning and delivering musculoskeletal care in the expanding older population and may help to guide future prevalence studies.

\section{Prevalence estimates}

Estimates for LSS with comorbid knee OA exhibited an increase in prevalence moving from combined to clinical to imaging knee OA definitions. The opposite pattern was observed in estimates of LSS and comorbid hip $\mathrm{OA}$, where combined hip OA definitions had the highest prevalence and imaging the lowest. This pattern is counterintuitive because imaging findings are a prerequisite of combined (imaging findings plus clinical symptoms) case definitions. Many people with imaging evidence of OA are clinically asymptomatic [16] and therefore we would expect the prevalence of combined imaging plus clinical definitions to be less prevalent than imaging-only definitions, as observed in the LSS with comorbid knee OA estimates.
An insufficient number of studies on index knee or hip OA and comorbid LSS prevented us from comparing prevalence patterns. However, in the two studies providing data on knee OA (combined case definitions) and comorbid LSS, a greater prevalence was observed for combined comorbid LSS than clinical comorbid LSS (54\% vs. $17 \%)$, suggesting a similarly counterintuitive pattern.

A meta-analysis by Pereira et al., [16] found the prevalence of radiographic hip OA was $15 \%$ versus $11 \%$ for symptomatic (combined radiographic and clinical definition) hip OA, which is different than the findings of our review (2\% imaging; 35\% combined). Conversely, the findings of our pooled analysis on LSS with comorbid knee OA follow the observed pattern found by Pereira et al., [16], where radiographic knee OA prevalence estimates were greater than symptomatic prevalence estimates ( $32 \%$ versus $21 \%$, respectively). In one study providing data on the prevalence of comorbid LSS in a knee OA sample primarily from secondary care settings, the prevalence of clinically-defined LSS $[17 \%$ (95\% CI 17-17\%)] [38] was lower than that observed in a recent meta-analysis of prevalence estimates of clinically-defined LSS in secondary care samples [29\% (95\% CI 22-36\%)] and mixed primary and secondary care samples [39\% (95\% 38-39\%)] [9]. The second study reporting the prevalence of comorbid LSS in a secondary care knee 


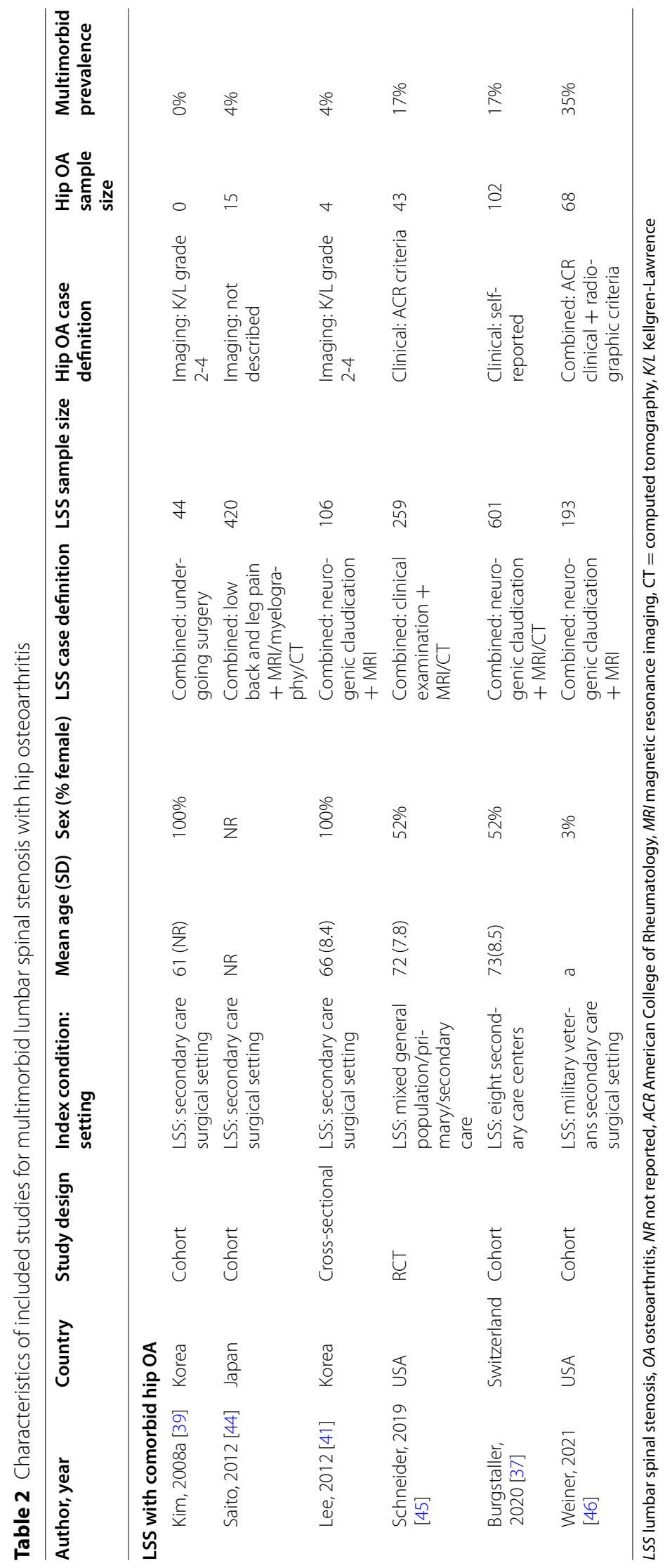




\begin{tabular}{lll} 
Author & Sample size & Prevalence $(95 \% \mathrm{Cl})$ \\
\hline $\begin{array}{l}\text { Combined Hip OA } \\
\text { Weiner } 2021\end{array}$ & 193 & \\
\end{tabular}

$\begin{array}{llll}\text { Clinical Hip OA } & & & \\ \text { Schneider 2019 } & 259 & 0.17(0.13,0.22) \\ \text { Burgstaller 2020 } & 601 & - & 0.17(0.14,0.20) \\ & & - & 0.17(0.14,0.19)\end{array}$

Imaging Hip OA

Kim 2008a

44

$0.00(0.00,0.08)$

Saito 2012

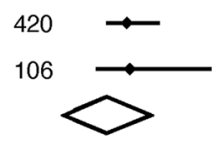

$0.04(0.02,0.06)$

Lee 2012

$0.04(0.01,0.09)$

$0.02(0.00,0.05)$

Fig. 3 Forest plot of results for combined lumbar spinal stenosis with comorbid hip osteoarthritis

OA sample [54\% (95\% CI 47-61\%)] [42] was much higher than reported in the LSS prevalence review.

\section{Risk of bias}

We were unable to assess the risk of bias impact on our prevalence estimates since all but one included study were rated as high risk of bias. The high risk of bias ratings are mainly a result of no studies having the aim of investigating the multimorbid prevalence. Therefore, the included studies may have adequate design for their primary purpose but suffer from a high risk of bias when appraised for the purpose of estimating prevalence. However, we did adapt a single-condition risk of bias tool [36] for the purposes of this study. Our ratings may therefore lack validity, but little guidance on risk of bias in multimorbidity prevalence studies is available. For example, multimorbidity prevalence reviews have not evaluated risk of bias [22] or have used reporting checklists as a surrogate measure [49]. To overcome this issue, we further modified a risk of bias tool used in a recent review on the prevalence of low back pain and co-occurring musculoskeletal pain sites [24] and in a review on the prevalence of LSS [9]. Future studies with low risk of bias might provide more clarity on the seemingly opposite prevalence patterns observed in this review. Further, consensus standards for studies of multimorbidity prevalence, and musculoskeletal-multimorbidity specifically, are first needed.

\section{Defining LSS and OA}

Different multimorbidity prevalence patterns may exist when using alternate case definitions for LSS. The multimorbidity prevalence pattern may also differ in samples where knee or hip OA is the index condition. Only two studies included data allowing for prevalence estimates of knee OA and comorbid LSS [38, 42] and we found no studies of hip OA and comorbid LSS. We were also unable to explore differences in prevalence estimates for varying clinical presentations of LSS, such as neurogenic claudication, radicular pain, or radiculopathy [31] as well as the impact differing levels of participant or clinical characteristics such as disease severity or stage, as this information was not consistently reported in the included studies.

No consensus on the exact features that define cases of LSS exists, whether based on imaging $[50,51]$ or clinical definitions. [52-54] The prevalence of multimorbid LSS and knee or hip OA relying on imaging-only case definitions may not be the best proxy, as imaging findings do not reliably represent the symptomatic experience of people with OA [17] or LSS [11]. In our review, only one included study specified the imaging findings considered to represent LSS [40]. The defining features of imagingbased knee and hip OA were more consistently reported in the included studies, but one study including imaging findings in the case definition did not describe the specific criteria used [44]. 


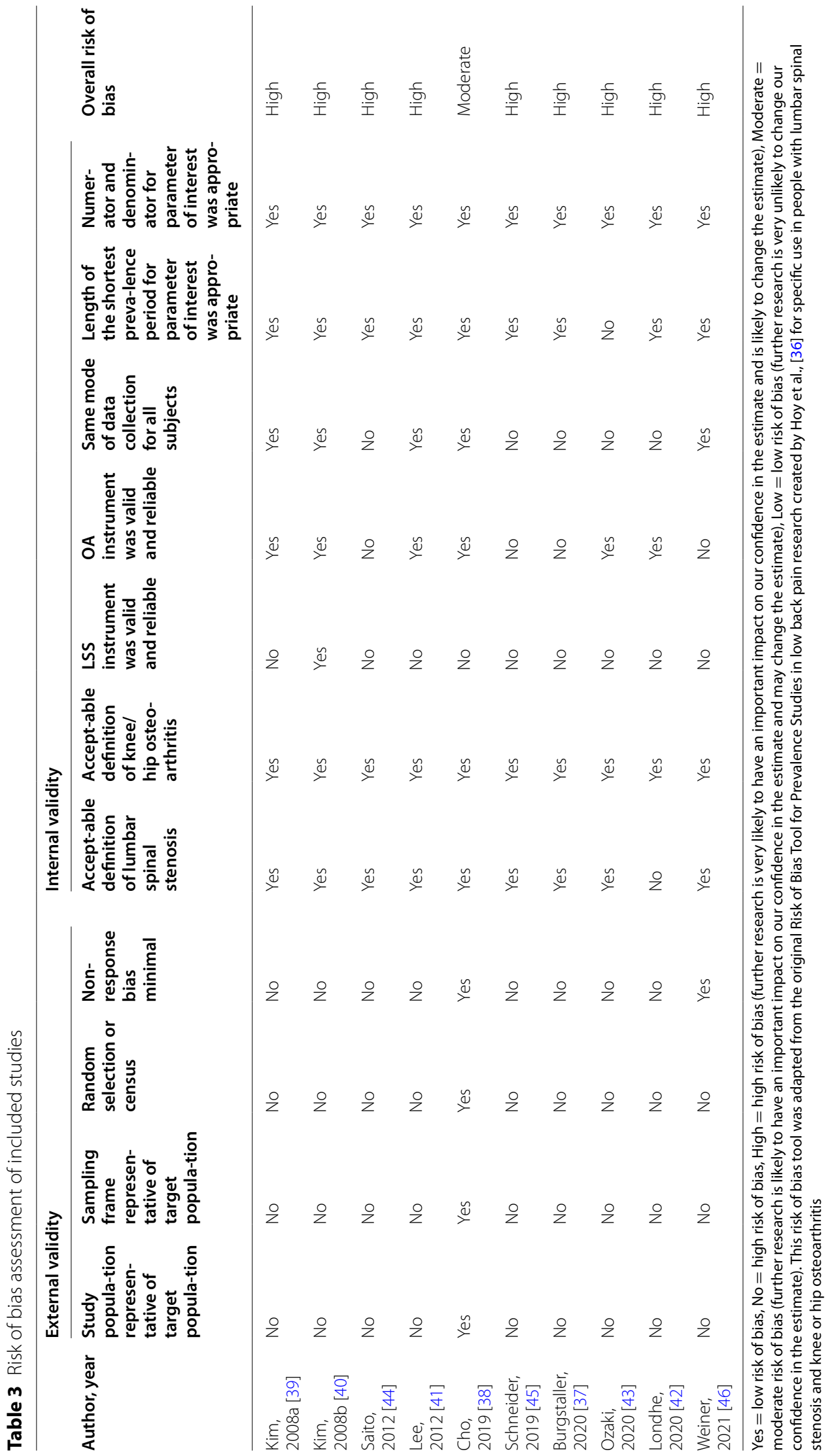


Combined clinical and imaging definitions likely represent the most reliable definition of LSS, however, when reported, variability existed in the exact imaging and clinical criteria. Many studies used a neurogenic claudication plus imaging findings on MRI, CT, or myelogram, but exact definitions were not reported. Likewise, studies with people undergoing surgery for LSS did not always report the surgical eligibility criteria.

\section{Influence of health care setting}

We were unable to assess the impact of health care setting on our results. Prevalence estimates of LSS [9], OA [16], and multimorbidity in general [2] differ according to the population of interest. Jensen et al. found that [9] the prevalence of LSS ranged from $11 \%$ in the general population to $29 \%$ in secondary care settings. All but two studies included in our review enrolled participants from secondary care settings [38, 45]. Although insurance claim data from all levels of health care was included, $73 \%$ of participants in the study by Cho et al., [38] received care in secondary care orthopedic and internal medicine departments. The second study by Schneider et al., [45] used a mixed sample of people from the general population, primary care, and secondary care, without providing data on the proportion of participants enrolled from each. Understanding the differences in prevalence estimates in diverse health care settings may help guide management priorities $[55,56]$.

\section{Implications for future studies}

Future studies should attempt to clarify the role chronic musculoskeletal conditions like LSS and OA play within the larger multimorbid burden faced by individuals and health systems. Musculoskeletal conditions increase one's risk of developing other non-communicable diseases [5], evidenced by the number of comorbidities in people with OA $[57,58]$ and LSS $[14,59]$. For example, Cho et al., [38] found Korean people with knee OA had comorbid: LSS (17\%), hypertension (45\%), diabetes (29\%), liver disease (20\%), depression (11\%), and other chronic conditions. Therefore, the prevalence of multimorbid LSS and knee and/or hip OA represents only a small portion of the larger impact experienced by those living with multiple chronic conditions $[4,60]$. Additionally, we only examined the prevalence of multimorbid LSS and knee or hip OA independently, but co-occurring patterns including both knee, hips, and the lumbar spine may be more informative. Future studies should also pay particular attention to how multimorbidity is defined and measured, as variable case definitions (including symptoms and/or risk factors) alter prevalence estimates for LSS [9], OA [16], and multimorbidity in general $[2,60,61]$.

\section{Limitations}

The main limitation of this review is the lack of original studies with a primary objective matching our review question. As a result, included studies were at a high risk of bias, but it is unclear what effect this may have on prevalence estimates. It was also difficult to assess the eligibility of articles based on titles and abstracts alone. We employed an overly sensitive approach to study screening, where any article mentioning "lumbar spinal stenosis" alone, "knee or hip OA and comorbidities", or multiple musculoskeletal conditions was screened in full text. Despite this approach, two included studies [37, 45] were not captured in the search. It is possible other published articles contain relevant data to this review but were not identified. Echoing recommendations from the wider multimorbidity literature [61], future reviews on this topic would benefit from the inclusion of a "multimorbidity" indexing term in electronic databases.

Heterogeneity in case definitions for LSS and lack of studies establishing a valid and reliable method of LSS assessment also limits our findings. Two studies included case definitions such as self-report of conditions and diagnostic codes, which were not easily classified into our pre-specified case definition framework. More available relevant studies would allow for sensitivity analyses to evaluate the assumptions made when classifying these case definitions. Finally, debate exists on the most suitable method of measuring multimorbidity. The method of simply counting the presence of conditions is supported in previous literature [2], but may be limited in comparison to definitions including symptoms and risk factors [61]. Future studies investigating multimorbid LSS and knee and hip OA would be greatly improved with the standardization of case definitions for LSS, OA, and multimorbidity.

\section{Conclusions}

This review has summarized the evidence for the cooccurrence of LSS with knee or hip OA. There is evidence that LSS with knee or hip OA appears to be common, but that estimates are uncertain as the prevalence ranged from 0 to $54 \%$ depending on the joint assessed and case definition. The variability in case definitions used for both LSS and OA, lack of studies on populations outside of secondary care settings, and high risk of bias prevents us from reporting firm estimates. Future high-quality studies on the prevalence of LSS with knee or hip OA are needed.

\section{Abbreviations}

LSS: Lumbar spinal stenosis; OA: Osteoarthritis; PRISMA: Preferred Reporting Items for Systematic Reviews and Meta-Analysis. 


\section{Supplementary Information}

The online version contains supplementary material available at https://doi. org/10.1186/s12891-022-05104-3.

\section{Additional file 1.}

Additional file 2.

Additional file 3.

\section{Acknowledgements}

Not applicable.

\section{Authors' contributions}

$J J Y, R K J, J H, E R, C A$, and CBJ conceived the study idea. JJY and CBJ developed the search strategy with assistance from a research librarian. JJY and CBJ performed the database searches. JJY and RKJ screened studies for inclusion. JJY and RKJ performed data extraction and risk of bias assessments. JJY, RKJ, and CBJ performed all analyses. All authors participated in the interpretation and formation of conclusions. JJY drafted the final report with critical revision and contribution from all authors. All authors give their approval for submission for publication of the final version of this systematic review article.

\section{Funding}

This study was supported by PhD funding for JJY from the Danish Foundation for Chiropractic Research and Post-graduate Education, Ontario Chiropractic Association, Canadian Memorial Chiropractic College, National Chiropractic Mutual Insurance Company Foundation, and the University of Southern Denmark.

\section{Availability of data and materials}

The datasets used and/or analysed during the current study are available from the corresponding author on reasonable request.

\section{Declarations}

Ethics approval and consent to participate

Not applicable.

\section{Consent for Publication}

Not applicable.

\section{Competing interests}

The authors declare that they have no competing interests.

\section{Author details}

${ }^{1}$ Center for Muscle and Joint Health, University of Southern Denmark, Campusvej 55, 5230 Odense M, Denmark. ${ }^{2}$ Research Division, Canadian Memorial Chiropractic College, 6100 Leslie Street, Toronto, Canada. ${ }^{3}$ Centre for Muscle and Joint Health, Department of Sports Science and Clinical Biomechanics, University of Southern Denmark, 55 Campusvej, DK-5230 Odense M, Denmark. ${ }^{4}$ Chiropractic Knowledge Hub, Odense M, Denmark. ${ }^{5}$ Rebecca MacDonald Centre for Arthritis and Autoimmune Diseases, Mount Sinai Hospital, Toronto, Canada. ${ }^{6}$ Institute for Health Policy, Management and Evaluation, University of Toronto, Toronto, Canada. ${ }^{7}$ Department of Physiotherapy and Occupational Therapy, Copenhagen University Hospital, Herlev and Gentofte, Copenhagen, Denmark.

Received: 29 June 2021 Accepted: 9 February 2022

Published online: 24 February 2022

\section{References}

1. Afshar S, Roderick PJ, Kowal P, Dimitrov BD, Hill AG. Global patterns of multimorbidity: a comparison of 28 countries using the world health surveys. In: Hoque MN, Pecotte B, McGehee MA, editors. Applied Demography and Public Health in the 21st Century. Springer; 2017. p.381-402.
2. Johnston MC, Crilly M, Black C, Prescott GJ, Mercer SW. Defining and measuring multimorbidity: a systematic review of systematic reviews. Eur J Public Health. 2019;29(1):182-9. https://doi.org/10.1093/eurpub/ cky098.

3. MacMahon S, Calverley P, Chaturvedi N, Chen Z, Corner L, Davies M, et al. Multimorbidity: a priority for global health research. The Academy of Medical Sciences. 2018. https://acmedsci.ac.uk/file-download/ 82222577.

4. Stewart M, Fortin M, Brown JB, Ryan BL, Pariser P, Charles J, et al. Patient-centred innovation for multimorbidity care: a mixed-methods, randomised trial and qualitative study of the patients' experience. $\mathrm{Br}$ J Gen Pract. 20201;71(705):e320-e330. https://doi.org/10.3399/bjgp2 $1 \times 714293$.

5. Williams A, Kamper SJ, Wiggers JH, O'Brien KM, Lee H, Wolfenden L, et al. Musculoskeletal conditions may increase the risk of chronic disease: a systematic review and meta-analysis of cohort studies. BMC Med. 2018;16(1):1-9. https://doi.org/10.1186/s12916-018-1151-2.

6. Briggs AM, Schneider CH, Slater H, Jordan JE, Parambath S, Young $J$ J, et al. Health systems strengthening to arrest the global disability burden: empirical development of prioritised components for a global strategy for improving musculoskeletal health. BMJ Glob Health. 2021;6(6):e006045. https://doi.org/10.1136/bmjgh-2021-006045.

7. Briggs AM, Valentijn PP, Thiyagarajan JA, de Carvalho IA. Elements of integrated care approaches for older people: a review of reviews. BMJ Open. 2018;8(4):e021194. https://doi.org/10.1136/bmjop en-2017-021194.

8. Deyo RA. Treatment of lumbar spinal stenosis: a balancing act. Spine J. 2010;10(7):625-7. https://doi.org/10.1016/j.spinee.2010.05.006.

9. Jensen RK, Jensen TS, Koes B, Hartvigsen J. Prevalence of lumbar spinal stenosis in general and clinical populations: a systematic review and meta-analysis. Eur Spine J. 2020:1-21. https://doi.org/10.1007/ s00586-020-06339-1.

10. Safiri S, Kolahi A-A, Smith E, Hill C, Bettampadi D, Mansournia MA, et al. Global, regional and national burden of osteoarthritis 1990-2017: a systematic analysis of the global burden of disease study 2017. Ann Rheum Dis. 2020;79(6):819-28. https://doi.org/10.1136/annrh eumdis-2019-216515.

11. Lurie J, Tomkins-Lane C. Management of lumbar spinal stenosis. BMJ. 2016;352 h6234. https://doi.org/10.1136/bmj.h6234.

12. Prather $\mathrm{H}$, van Dillen L. Links between the hip and the lumbar spine (hip spine syndrome) as they relate to clinical decision making for patients with Lumbopelvic pain. PM R. 2019;11:S64-S72. https://doi.org/10.1002/ pmrj.12187.

13. Goode AP, Cleveland RJ, George SZ, Kraus VB, Schwartz TA, Gracely RH, et al. Different phenotypes of osteoarthritis in the lumbar spine reflected by demographic and clinical characteristics: the Johnston County Osteoarthritis Project. Arthritis Care Res. 2020;72(7):974-81. https://doi.org/10. 1002/acr.23918.

14. Ferrero E, Lonjon G, Bouyer B, Sabourin M, Ould-Slimane M, Guigui P. Influence of comorbidities on patients reported outcomes in degenerative lumbar spinal stenosis. Orthop Traumatol Surg Res. 2018;104(7):1031-6. https://doi.org/10.1016/j.otsr.2018.07.012.

15. Held U, Burgstaller JM, Wertli MM, Pichierri G, Winklhofer S, Brunner F, et al. Prognostic function to estimate the probability of meaningful clinical improvement after surgery-Results of a prospective multicenter observational cohort study on patients with lumbar spinal stenosis. PloS One. 2018;13(11):e0207126. https://doi.org/10.1371/journal.pone.0207126.

16. Pereira D, Peleteiro B, Araujo J, Branco J, Santos RA, Ramos E. The effect of osteoarthritis definition on prevalence and incidence estimates: a systematic review. Osteoarthritis Cartilage. 2011;19(11):1270-85. https:// doi.org/10.1016/j.joca.2011.08.009.

17. Sharma L. Osteoarthritis of the knee. N Engl J Med. 2021;384(1):51-9. https://doi.org/10.1056/NEJMcp1903768.

18. Bayliss EA, Bayliss MS, Ware JE, Steiner JF. Predicting declines in physical function in persons with multiple chronic medical conditions: what we can learn from the medical problem list. Health Qual Life Outcomes. 2004;2:47. https://doi.org/10.1186/1477-7525-2-47.

19. Fortin M, Lapointe L, Hudon C, Vanasse A, Ntetu AL, Maltais D. Multimorbidity and quality of life in primary care: a systematic review. Health Qual Life Outcomes. 2004;2:51. https://doi.org/10.1186/1477-7525-2-51. 
20. Ryan A, Wallace E, O'Hara P, Smith SM. Multimorbidity and functional decline in community-dwelling adults: a systematic review. Health Qual Life Outcomes. 2015;13:168. https://doi.org/10.1186/s12955-015-0355-9.

21. Butera KA, Roff SR, Buford TW, Cruz-Almeida Y. The impact of multisite pain on functional outcomes in older adults: biopsychosocial considerations. J Pain Res. 2019;12:1115-25. https://doi.org/10.2147/JPR.S192755.

22. De Luca K, Wong A, Eklund A, Fernandez M, Byles JE, Parkinson L, et al. Multisite joint pain in older Australian women is associated with poorer psychosocial health and greater medication use. Chiropr Man Therap. 2019;27:8. https://doi.org/10.1186/s12998-018-0224-9.

23. Hartvigsen J, Natvig B, Ferreira M. Is it all about a pain in the back? Best Pract Res Clin Rheumatol. 2013;27(5):613-23. https://doi.org/10.1016/j. berh.2013.09.008.

24. Øverås CK, Johansson MS, de Campos TF, Ferreira ML, Natvig B, Mork PJ, et al. Distribution and prevalence of musculoskeletal pain co-occurring with persistent low back pain: a systematic review. BMC Musculoskelet Disord. 2021;22(1):91. https://doi.org/10.1186/s12891-020-03893-z.

25. Gullo TR, Golightly YM, Cleveland RJ, Renner JB, Callahan LF, Jordan JM, et al. Defining multiple joint osteoarthritis, its frequency and impact in a community-based cohort. Semin Arthritis Rheum. 2019;48(6):950-7. https://doi.org/j.semarthrit.2018.10.001.

26. Perruccio AV, Power JD, Evans HMK, Mahomed SR, Gandhi R, Mahomed $\mathrm{NN}$, et al. Multiple joint involvement in total knee replacement for osteoarthritis: effects on patient-reported outcomes. Arthritis Care Res. 2012;64(6):838-46. https://doi.org/10.1002/acr.21629.

27. Perruccio AV, Power JD, Yip C, Badley EM, Canizares M, Rampersaud YR. The impact of multijoint symptoms on patient-reported disability following surgery for lumbar spine osteoarthritis. Spine J. 2021;21(1):80-9. https://doi.org/10.1016/j.spinee.2020.08.005.

28. Young JJ, Hartvigsen J, Jensen RK, Roos EM, Ammendolia C, Juhl CB. Prevalence of multimorbid degenerative lumbar spinal stenosis with knee and/or hip osteoarthritis: protocol for a systematic review and meta-analysis. Syst Rev. 2020;9(1):232. https://doi.org/10.1186/ s13643-020-01478-4.

29. Higgins JPT, Thomas J, Chandler J, Cumpston M, LiT, Page MJ, et al. Cochrane handbook for systematic reviews of interventions: John Wiley \& Sons; 2019.

30. Page MJ, MCKenzie JE, Bossuyt PM, Boutron I, Hoffmann TC, Mulrow CD, et al. The PRISMA 2020 statement: an updated guideline for reporting systematic reviews. BMJ. 2021;372.n71. https://doi.org/10.1136/bmj.n71.

31. Suri P, Rainville J, Kalichman L, Katz JN. Does this older adult with lower extremity pain have the clinical syndrome of lumbar spinal stenosis? JAMA. 2010;304(23):2628-36. https://doi.org/10.1001/jama.2010.1833.

32. Ammendolia C, Stuber KJ, Rok E, Rampersaud R, Kennedy CA, Pennick V, et al. Nonoperative treatment for lumbar spinal stenosis with neurogenic claudication. Cochrane Database Syst Rev. 2013;(8):CD010712. https://doi. org/10.1002/14651858.CD010712.

33. Fransen M, McConnell S, Harmer AR, Van der Esch M, Simic M, Bennell $\mathrm{KL}$. Exercise for osteoarthritis of the knee. Cochrane Database Syst Rev. 2015;1:CD004376. https://doi.org/10.1002/14651858.CD004376.pub3.

34. Fransen M, McConnell S, Hernandez-Molina G, Reichenbach S. Exercise for osteoarthritis of the hip. Cochrane Database Syst Rev. 2014:4:CD007912. https://doi.org/10.1002/14651858.CD007912.pub2.

35. Cohen J. A coefficient of agreement for nominal scales. Educ Psychol Meas. 1960:20(1):37-46. https://doi.org/10.1177/001316446002000104.

36. Hoy D, Brooks P, Woolf A, Blyth F, March L, Bain C, et al. Assessing risk of bias in prevalence studies: modification of an existing tool and evidence of interrater agreement. J Clin Epidemiol. 2012;65(9):934-9. https://doi. org/10.1016/j.jclinepi.2011.11.014

37. Burgstaller JM, Steurer J, Gravestock I, Brunner F, Fekete TF, Pichierri G, et al. Long-term results after surgical or nonsurgical treatment in patients with degenerative lumbar spinal stenosis: a prospective multicenter study. Spine. 2020;45(15):1030-8. https://doi.org/10.1097/BRS.00000 00000003457.

38. Cho S-K, Jung S-Y, Choi S, Im SG, Kim H, Choi WS, et al. Factors related to the use of opioids as early treatment in patients with knee osteoarthritis. Arthritis Res Ther. 2019;21(1):222. https://doi.org/10.1186/ s13075-019-2004-X

39. Kim H-J, Kim S-G, Lee H-M, Kim H-S, Moon E-S, Park J-O, et al. Risk factors associated with the halo phenomenon after lumbar fusion surgery and its clinical significance. Asian Spine J. 2008;2(1):22-6. https://doi.org/10. 4184/asj.2008.2.1.22.

40. Kim H-J, Lee H-M, Kim H-S, Park J-O, Moon E-S, Park H, et al. Bone metabolism in postmenopausal women with lumbar spinal stenosis: analysis of bone mineral density and bone turnover markers. Spine. 2008;33(22):2435-9. https://doi.org/10.1097/BRS.0b013e3181829fca.

41. Lee BH, Moon SH, Kim H-J, Lee HM, Kim TH. Osteoporotic profiles in elderly patients with symptomatic lumbar spinal canal stenosis. Indian J Orthop. 2012:46(3):279-84. https://doi.org/10.4103/0019-5413.96379.

42. Londhe SB, Shah RV, Patwardhan M, Doshi AP, Londhe SS, Subhedar K, et al. Study of patients with bilateral knee osteoarthritis undergoing total knee replacement procedure with coexisting lumbar spondylosis symptoms. Asian Spine J. 2020. https://doi.org/10.31616/asj.2020.0279.

43. Ozaki M, Fujita N, Miyamoto A, Suzuki S, Tsuji O, Nagoshi N, et al. Impact of knee osteoarthritis on surgical outcomes of lumbar spinal canal stenosis. J Neurosurg Spine. 2019;32(5):710-5. https://doi.org/10.3171/2019.10. SPINE19886.

44. Saito J, Ohtori S, Kishida S, Nakamura J, Takeshita M, Shigemura T, et al. Difficulty of diagnosing the origin of lower leg pain in patients with both lumbar spinal stenosis and hip joint osteoarthritis. Spine. 2012;37(25):2089-93. https://doi.org/10.1097/BRS.0b013e31825d213d.

45. Schneider MJ, Ammendolia C, Murphy DR, Glick RM, Hile E, Tudorascu DL, et al. Comparative clinical effectiveness of nonsurgical treatment methods in patients with lumbar spinal stenosis: a randomized clinical trial. JAMA Netw Open. 2019;2(1):e186828-e. https://doi.org/10.1001/jaman etworkopen.2018.6828.

46. Weiner DK, Holloway K, Levin E, Keyserling H, Epstein F, Monaco E, et al. Identifying biopsychosocial factors that impact decompressive laminectomy outcomes in veterans with lumbar spinal stenosis: a prospective cohort study. Pain. 2021;162(3):835-45. https://doi.org/10.1097/j.pain. 0000000000002072.

47. McNamara MJ, Barrett KG, Christie MJ, Spengler DM. Lumbar spinal stenosis and lower extremity arthroplasty. J Arthroplasty. 1993;8(3):273-7. https://doi.org/10.1097/j.pain.0000000000002072.

48. Whitman JM, Flynn TW, Childs JD, Wainner RS, Gill HE, Ryder MG, et al. A comparison between two physical therapy treatment programs for patients with lumbar spinal stenosis: a randomized clinical trial. Spine. 2006;31(22):2541-9. https://doi.org/10.1097/01.brs.0000241136.98159.8c.

49. Fortin M, Stewart M, Poitras M-E, Almirall J, Maddocks H. A systematic review of prevalence studies on multimorbidity: toward a more uniform methodology. Ann Fam Med. 2012;10(2):142-51. https://doi.org/10.1370/ afm.1337.

50. Andreisek, G, Imhof M, Wertli M, Winklhofer SFX, Pfirrmann CWA, et al. A systematic review of semiquantitative and qualitative radiologic criteria for the diagnosis of lumbar spinal stenosis. Am J Roentgenol. 2013;201(5):735-46. https://doi.org/10.2214/AJR.12.10163.

51. Andreisek G, Deyo RA, Jarvik JG, Porchet F, Winklhofer SFX, Stteurer J, Hodler J, et al. Consensus conference on core radiological parameters to describe lumbar stenosis - an initiative for structured reporting. Eur Radiol. 2014;24(12):3224-32. https://doi.org/10.1007/s00330-014-3346-z.

52. Tomkins-Lane C, Melloh M, Lurie J, Smuck M, Battié MC, Freeman B, et al. Consensus on the clinical diagnosis of lumbar spinal stenosis: results of an international Delphi study. Spine. 2016;41(15):1239-46. https://doi.org/ 10.1097/BRS.0000000000001476.

53. Genevay S, Atlas SJ, Katz JN.Variation in eligibility criteria from studies of radiculopathy due to a herniated disc and of neurogenic claudication due to lumbar spinal stenosis: a structured literature review. Spine. 2010;35(7):80311. https://doi.org/10.1097/BRS.0b013e3181bc9454.

54. de Schepper EIT, Overdevest GM, Suri P, Peul WC, Oei EHG, Koes BW, et al. Diagnosis of lumbar spinal stenosis: an updated systematic review of the accuracy of diagnostic tests. Spine, 2013;38(8):469-81. https://doi.org/10. 1097/BRS.0b013e31828935ac.

55. National Insititue for Health and Care Excellence. Multimorbidity: clinical assessment and management. National Guideline Centre (UK). 2016;NG56. https://www.nice.org.uk/guidance/ng56.

56. Smith S, Wallace E, Clyne B, Boland F, Fortin M. Interventions for improving outcomes in patients with multimorbidity in primary care and community setting: a systematic review. Syst Rev. 2021. https://doi.org/ 10.21203/rs.3.rs-144669/v1.

57. Calders P, Van Ginckel A. Presence of comorbidities and prognosis of clinical symptoms in knee and/or hip osteoarthritis: a systematic review and 
meta-analysis. Semin Arthritis Rheum. 2018;47:805-13. https://doi.org/10. 1016/j.semarthrit.2017.10.016.

58. Swain S, Sarmanova A, Coupland C, Doherty M, Zhang W. Comorbidities in Osteoarthritis: A systematic review and meta-analysis of observational studies. Arthritis Care Res. 2020;72(7):991-1000. https://doi.org/10.1002/ acr.24008.

59. Battié MC, Jones CA, Schopflocher DP, Hu RW. Health-related quality of life and comorbidities associated with lumbar spinal stenosis. Spine J. 2012;12(3):189-95. https://doi.org/10.1016/j.spinee.2011.11.009.

60. Griffith LE, Gilsing A, Mangin D, Patterson C, van den Heuvel E, Sohel N, et al. Multimorbidity frameworks impact prevalence and relationships with patient-important outcomes. J Am Geriatr Soc. 2019;67(8):1632-40. https://doi.org/10.1111/jgs.15921.

61. De Schepper EIT, et al. Diagnosis of lumbar spinal stenosis: an updated systematic review of the accuracy of diagnostic tests. Spine. 2013;38(8):E469-81.

62. Excellence, N.I.f.H.a.C., Multimorbidity: clinical assessment and management. 2016. NG56

63. Smith, S., et al., Interventions for Improving Outcomes in Patients With Multimorbidity in Primary Care and Community Setting: A Systematic Review. 2021

64. Willadsen TG, Bebe A, Køster-Rasmussen R, Jarbøl DE, Guassora AD, Waldorff FB, et al. The role of diseases, risk factors and symptoms in the definition of multimorbidity-a systematic review. Scand J Prim Health Care. 2016;34(2):112-21. https://doi.org/10.3109/02813432.2016.1153242.

\section{Publisher's Note}

Springer Nature remains neutral with regard to jurisdictional claims in published maps and institutional affiliations.

- fast, convenient online submission

- thorough peer review by experienced researchers in your field

- rapid publication on acceptance

- support for research data, including large and complex data types

- gold Open Access which fosters wider collaboration and increased citations

- maximum visibility for your research: over $100 \mathrm{M}$ website views per year

At BMC, research is always in progress.

Learn more biomedcentral.com/submissions 